\title{
General Cyclopropane Assembly via Enantioselective Transfer of a Redox-Active Carbene to Aliphatic Olefins
}

\author{
Marc Montesinos-Magraner, ${ }^{[a] \ddagger}$ Matteo Costantini, ${ }^{[a] \ddagger}$ Rodrigo Ramírez-Contreras, ${ }^{[a]}$ Michael E. \\ Muratore ${ }^{[b]}$ Magnus J. Johansson ${ }^{[b]}$ and Abraham Mendoza*[a]
}

\begin{abstract}
Asymmetric cyclopropane synthesis currently requires bespoke strategies, methods, substrates and reagents, even when targeting similar compounds. This slows down discovery and limits its available chemical space. Here we introduce a practical and versatile diazocompound, and we demonstrate its performance in the first unified asymmetric synthesis of functionalized cyclopropanes. We found that the redox-active leaving group in this reagent enhances the reactivity and selectivity of geminal carbene transfer. This effect allowed for the asymmetric cyclopropanation of various olefins including unfunctionalized aliphatic alkenes, that enables the 3-step total synthesis of (-)-dictyopterene A. This unified synthetic approach delivers high enantioselectivities that are independent of the stereoelectronic properties of the functional groups transferred. Our results demonstrate that orthogonallydifferentiated diazocompounds are viable and advantageous equivalents of single-carbon chirons.
\end{abstract}

Cyclopropanes have attracted the attention of chemists for decades. The high strain and unique bonding of these carbocycles display enhanced conformational control and oxidative resistance that have been exploited in organic synthesis, ${ }^{[1 \mathrm{a}]}$ asymmetric catalysis ${ }^{[1 \mathrm{~b}]}$ and medicinal chemistry. ${ }^{[1 \mathrm{c}]}$ Cyclopropanes are common in advanced total syntheses both as structural elements in challenging natural products, ${ }^{[1 \mathrm{~d}]}$ and as enabling instruments to drive creative disconnections. ${ }^{[1 \mathrm{e}]}$ Different asymmetric syntheses of functionalized cyclopropanes 1 have thus been developed (Scheme 1a). ${ }^{[2],[3]}$ However, similar targets with different peripheral functionality still require discrete strategies based on specific reagents, catalysts, and substrates that are elaborated over several steps from raw sources. The key enantioselective step on these routes are often based on allylic alcohols $2,{ }^{[4]} 1,1$-disubstituted cyclopropenes $3,{ }^{[5]}$ or electron-deficient alkenes $4{ }^{[6]}$ The asymmetric cyclopropanation of unfunctionalized olefins $(5$; i.e. electron-rich and styrenes) with diazoesters, has been extensively developed with metal-, ${ }^{[7]}$ and bio-catalysts. ${ }^{[8]}$ However, common aliphatic alkenes ${ }^{[9]}$ are, despite elegant approaches, ${ }^{[7 b, 7 c, 8 c, 8 d]}$ still problematic due to their low nucleophilicity, and the flexibility and weak dispersive

[a] Dr. M. Montesinos-Magraner, ${ }^{\ddagger}$ M. Costantini, ${ }^{\ddagger}$ Dr. R. RamírezContreras, Dr. A. Mendoza

Dept. of Organic Chemistry, Stockholm University Arrhenius Laboratory 10691 Stockholm (Sweden)

E-mail: abraham.mendoza@su.se

[b] Dr. M. E. Muratore, Dr. M. J. Johansson Cardiovascular, Renal and Metabolism IMED Biotech Unit AstraZeneca Gothenburg

Pepparedsleden 1, 43183 Mölndal (Sweden)

[†] These authors contributed equally

Supporting information for this article is given via a link at the end of the document. interactions of their alkyl substituent. ${ }^{[7 b-i, 10]}$ Importantly, many interesting functionalized carbenes 6-12 (FG-C-H), such as alkyl-, ${ }^{[11 a,}{ }^{11 b]}$ hydroxy-, ${ }^{[11 c]}$ amino-, ${ }^{[11 d]}$ boryl-, ${ }^{[11 e]}$ selenyl-, ${ }^{[11 f]}$ heteroaryl-, ${ }^{[11 \mathrm{~g}]}$ or alkenyl-methylidenes ${ }^{[11 \mathrm{~h}]}$ are too unstable to engage in cyclopropanation reactions. As a result, the derived

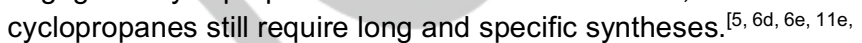
12]

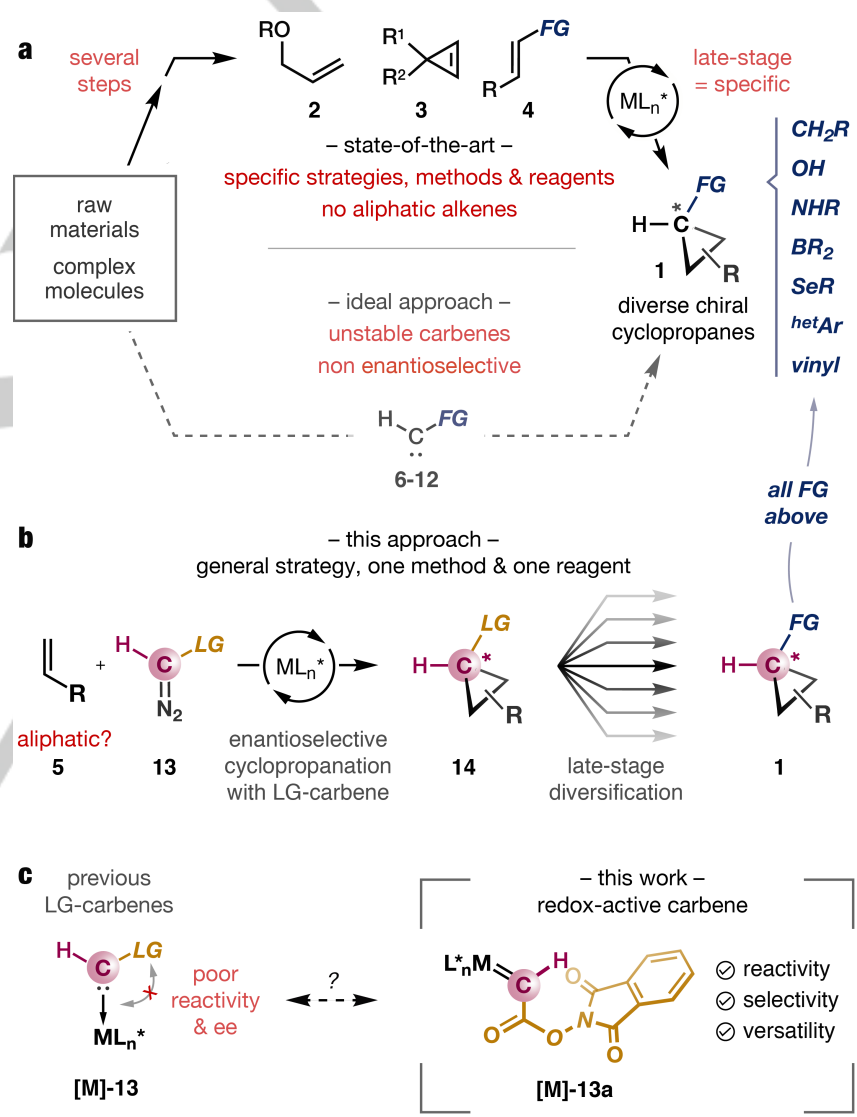

Scheme 1. State-of-the-art, concept and challenges towards the unified assembly of diverse chiral cyclopropanes. ${ }^{\text {het }} \mathrm{Ar}=$ heteroaryl, $\mathrm{FG}=$ functional group.

We conceived a unified strategy ${ }^{[13]}$ to comprehensively address the formal asymmetric transfer of carbenes 6-12 to alkene feedstocks 5 through a two-stage process (Scheme $1 \mathrm{~b}$ ): [1] enantioselective cyclopropanation of 5 with a diazocompound featuring a leaving group (13); and [2] late-stage diversification of the resulting intermediates 14 . This approach would produce chiral products with identical enantioselectivity regardless of their specific functionality, while simplifying the optimization of the enantioselective cyclopropanation by using a single carbene 
precursor. However, leaving group-functionalized carbenes [M]13 (Scheme 1c) are known to be poorly reactive, nonenantioselective, and their precursors tend to undergo substitution prior to the carbene transfer. ${ }^{[14]}$ We envisioned that redox-active carbenes [M]-13a (Scheme 1c) - containing an $\mathrm{N}$ hydroxyphthalimide (NHPI) ester leaving group - could become equivalent to all the distinct carbenes $\mathbf{6 - 1 2}$, due to the dual capacity of NHPI esters to act as acyl electrophile ${ }^{[15]}$ and radical precursors. Their vividly expanding $\mathrm{C}-\mathrm{C},{ }^{[16]} \mathrm{C}-\mathrm{N},{ }^{[17]} \mathrm{C}-\mathrm{O}^{[18]}$ or $\mathrm{C}-$ $\mathrm{B}^{[19]}$ bond forming reactions, are well beyond the reach of current divergent strategies towards chiral cyclopropanes. ${ }^{[20,21 a]}$ Despite their versatility, the orthogonality of NHPI esters to metal-carbenes was unknown, and a logical concern when considering their facile irreversible fragmentation upon activation with various transition metals and/or visible light. ${ }^{[16-19]}$

After extensive experimentation, we found that the designed $\mathrm{N}$-hydroxyphthalimidoyl diazoacetate reagent (NHPI-DA, 13a; Scheme $2 a)^{[22]}$ can be isolated in gram-amounts as a crystalline solid that is bench-stable for months. NHPI-DA (13a) does not require solution storage, and thus allows for easier handling. It also displays higher stability than the benchmark ethyl diazoacetate, as evidenced by DSC (EDA, 15a; Scheme 2b). Initial evaluation of NHPI-DA (13a) in cyclopropanation reactions using common rhodium, copper and palladium catalysts ${ }^{[2]}$ displayed low efficiency and selectivity (Table S3), as anticipated for this challenging transformation (vide supra). Fortunately, we discovered that NHPI-DA (13a) is effective in combination with the electron-rich metallacyclic ruthenium catalyst RuL1 ${ }^{[21]}$ (Scheme 2c), displaying a remarkable cyclopropanation selectivity over the competing dimerization pathway (16-18/19). We compared this intrinsic selectivity against various conventional diazo reagents with different steric and electronic properties (15a-f). For this study, we used equimolar conditions, fast addition of 13a,15a-f, and model olefins 5a-c with distinct nucleophilicities ( $N ; ;^{[23]}$ Scheme $2 d$ ), to minimize substrate-specific bias. It was found that NHPI-DA (13a) outperforms diazocompounds $15 a-f$ using styrene (5a, $N=$ +1.70 ), and this trend is importantly accentuated with more challenging olefins, such as 1-hexene $(5 \mathbf{b}$, $N=-2.77)$ and vinyltrimethylsilane $(5 \mathbf{c}, N<-1.46)$. This effect can be rationalized by the stronger electron-withdrawing effect of the ester moiety in $\mathbf{1 3 a}$, which allows less reactive olefins to effectively compete for the electrophilic carbene Int-A (Scheme 2c).

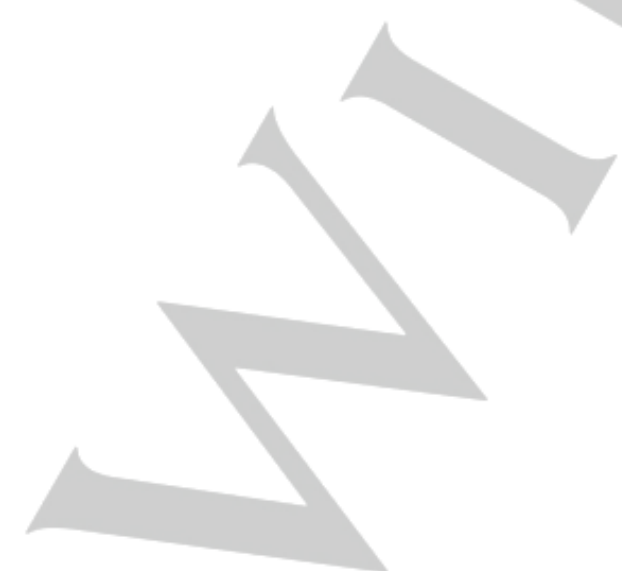

a
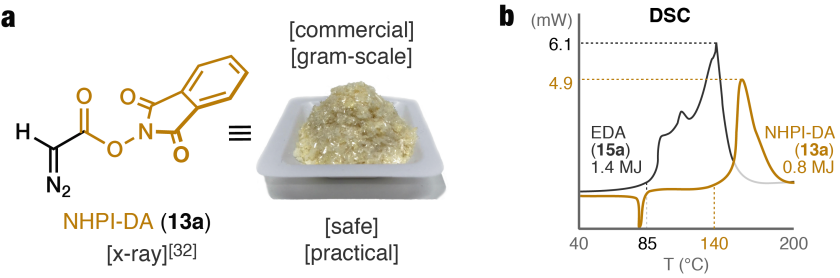

c
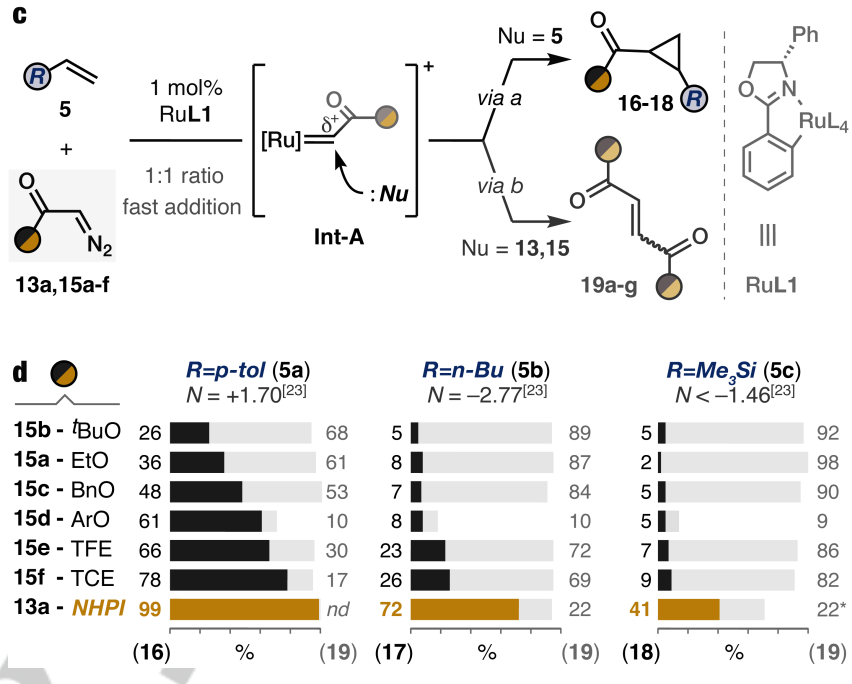

Scheme 2. Properties and performance of NHPI-DA (13a). Conditions: 5 (0.1 mmol), RuL1 (1 mol\%), 13,15 (0.1 mmol; $1 \mathrm{~s}$ addition time), $\mathrm{CH}_{2} \mathrm{Cl}_{2}, 0{ }^{\circ} \mathrm{C}$ $\mathrm{DSC}=$ differential scanning calorimetry, $\mathrm{EDA}=$ ethyl diazoacetate, $\mathrm{Bn}=$ benzyl, $\mathrm{ArO}=$ 2,6-di-(tert-butyl)-4-methylphenoxy, TFE = 2,2,2-trifluoroethoxy, TCE $=$ 2,2,2-trichloroethoxy.

Although RuL1 is a competent catalyst in asymmetric cyclopropanation, ${ }^{[21]}$ we have found that NHPI-DA (13a) expands its scope into an unprecedentedly wide selection of alkenes with surprisingly high enantioselectivities (Scheme 3). ${ }^{[2]}$ Functionalized styrenes with various substitution patterns lead, without slow addition, to redox-active cyclopropanes $16,20-28$ in excellent yields, and with high diastereo- and enantioselectivities. Different nucleophilic enol ethers smoothly produce push-pull products $\mathbf{2 9 , 3 0}$, even in the presence of a primary alcohol (no O-H insertion observed). Various types of enamines (31-33) or an allylic silane (34), are also efficiently cyclopropanated. Importantly, weakly nucleophilic aliphatic olefins with flexible alkyl substituents, ${ }^{[7,10]}$ furnish cyclopropanes 17,35 with high diastereo- and enantioselectivity under standard conditions. We explored the functional group tolerance of this method in these series using substrates equipped with a pendant arene (36), ketone (37), chloride (38), Weinreb amide (39), alcohol derivative (40) and alkyne (41), observing high enantioselectivity in all cases. Likewise, methylenecycloalkanes produce the interesting spiro-cyclopropane building blocks $\mathbf{4 2 , 4 3}$, and protected amines (44) were also tolerated. As far as we are aware, the NHPI-DA / RuL1 combination is the simplest homogeneous system to achieve highly enantioselective cyclopropanation on a wide range of aliphatic alkenes. ${ }^{[7,8,10]}$ Natural aliphatic olefin feedstocks offer an opportunity to explore its performance in complex settings. Complete discrimination between olefins of different nucleophilicity (including enones and 1,3-dienes) 


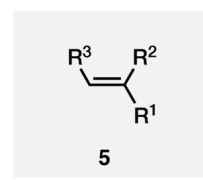

abundant olefins

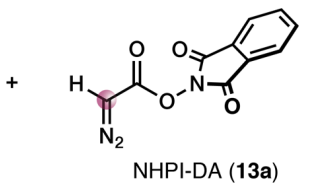

(1.2 equiv)

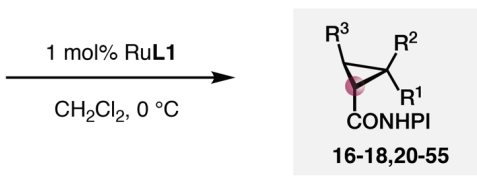

enantiopure cyclopropanes

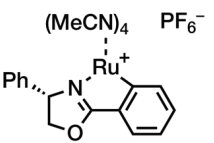

(S)-RuL1

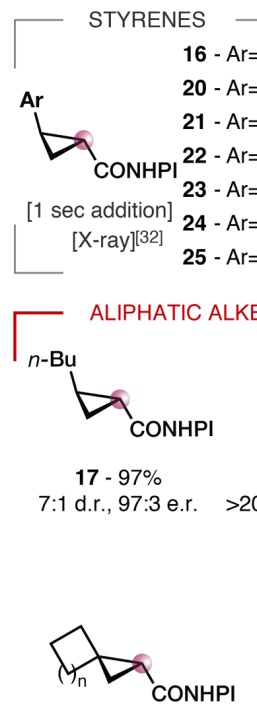

42 - $n=198 \%, 99: 1$ e.r. $43-n=399 \%, 99: 1$ e.r.

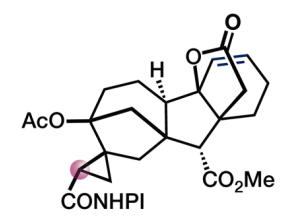

47 - $83 \%$ $>20: 1 \mathrm{rr}, 8.5: 1 \mathrm{~d} . \mathrm{r}[\mathrm{c}]$

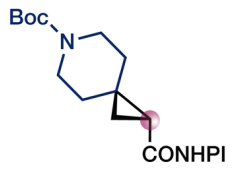

$44-88 \%$ 98:2 e.r.

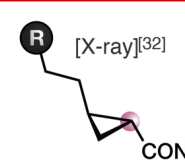

CON
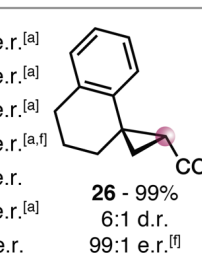

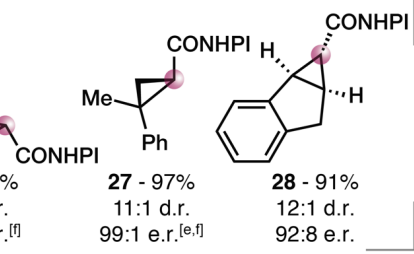

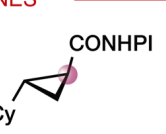

$35-93 \%$
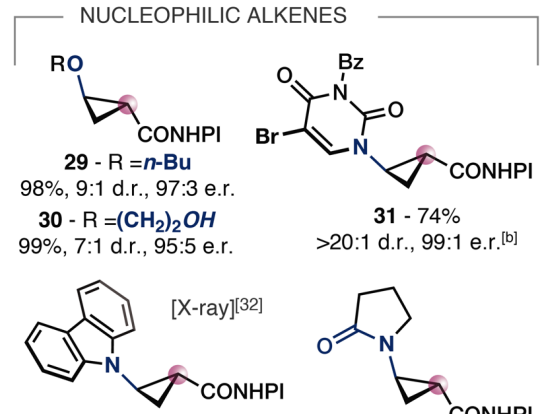

$31-74 \%$ $>20: 1$ d.r., $99: 1$ e.r. ${ }^{[b]}$<smiles>O=C1CC1N1CCCC1=O</smiles>
$\begin{array}{cc}32-79 \% & 33-96 \% \\ 20: 1 \text { d.r., } 98: 2 \text { e.r. }{ }^{[f]} & 1.7: 1 \text { d.r., } 99: 1 \text { e.r }\end{array}$ $>20: 1$ d.r., $98: 2$ e.r.

$45-97 \%$ $>20: 1$ r.r., $16: 1$ d.r. ${ }^{[e]}$

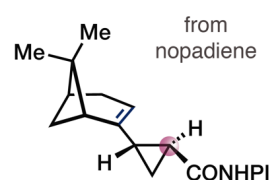

$46-92 \%$ $>20: 1 \mathrm{rr},>20: 1$ d.r. ${ }^{[e]}$

\section{from} acid

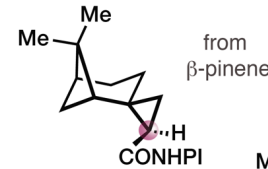

$48-99 \%,>20 \cdot 1$ d ${ }^{[b]}$

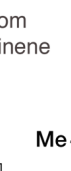

\section{from}

rebaudioside $A$

(in Stevia extract)

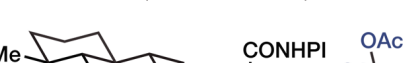
CONHPI OAC<smiles>C1C2CC1C21CC1</smiles>

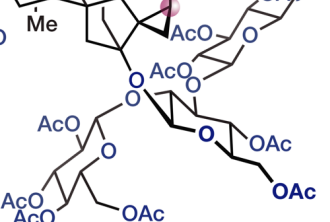

$51-59 \%, 6: 1$ d.r. ${ }^{[c, e]}$

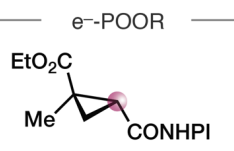

$53-72 \%$ 12:1 d.r., $94: 6$ e.r. $[\mathrm{b}, \mathrm{fl}$

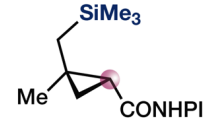

$34-99 \%$ 1.2:1 d.r., $99: 1$ e.r.

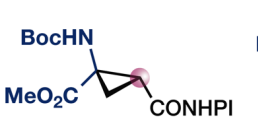

$\mathrm{R}^{\prime} \mathrm{R}_{2} \mathrm{Si} \mathrm{C}_{\mathrm{CONHPI}}$

$18-R^{\prime}=R=M e, 63 \%$ $>20: 1$ d.r., $98: 2$ e.r.

$54-63 \% \quad 55-R^{\prime}=P h, R=M e, 63 \%$ 4:1 d.r., $90: 10$ e.r. ${ }^{[b]} \quad>20: 1$ d.r., $98: 2$ e.r.
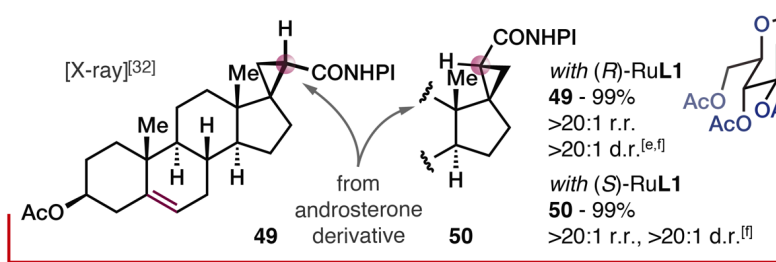

$50-99 \%$
$>20: 1$ r.r., $>20: 1$ d.r. ${ }^{[f]}$

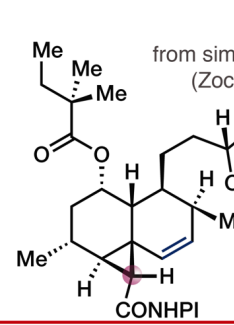

$52-71 \%$ $>20: 1$ d.r. ${ }^{[d, e]}$

Scheme 3. Scope of the enantioselective cyclopropanation with NHPI-DA (13a). Conditions: 5 (1.0 equiv), $13 a$ (1.2 equiv, 40 min addition time), ( $)$ )-RuL1 (1

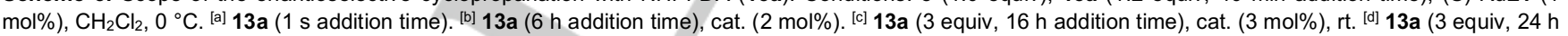
addition time), cat. $(5 \mathrm{~mol} \%)$, rt. ${ }^{[e]}$ Cat. $(R)$-RuL1. ${ }^{\left[{ }^{f]}\right]}$ Absolute configuration confirmed. See SI for experimental details. ${ }^{[32]} \mathrm{Bz}={ }^{\mathrm{benzoyl}}$, TIPS $=$ triisopropylsilyl, Boc $=$ tert-butyloxycarbonyl, Ac $=$ acetyl.

enables the selective modification of carvone, nopadiene, and gibberellic acid (45-47). Hindered olefins in the pinane (48) and steroid (49-50) frameworks also undergo smooth cyclopropanations. In the latter, either enantiomer of the catalyst RuL1 can be used to access alternative diastereomers 49 and 50 with complete facial discrimination. This system is also primed for late-stage functionalization of macromolecular natural glycosides and pharmaceuticals that would be problematic with existing biocatalysts, ${ }^{[8]}$ like rebaudioside $A$ obtained from a Stevia extract (51) and the cholesterol-regulating drug simvastatin (Zocor $®)$ (52). Electron-poor olefins produce the differentiated bis-carboxylate 53 and the cyclopropyl amino acid derivative 54. Silylcyclopropanes $\mathbf{1 8 , 5 5}$ are also obtained with high diastereo- and enantioselectivity from vinylsilanes, despite their high steric bulk.
We explored the synthetic potential of enantioenriched redox-active cyclopropane scaffolds derived from the representative olefins 5d,e (Scheme 4a). Upon decarboxylation, these are versatile precursors of catecholboronates ${ }^{[19 b]}$ that can be readily converted into pinacolboronates 56-57 or oxidized to cyclopropanols 58-59. Radical decarboxylative alkylation $(\mathbf{6 0}, 61)$ and selenation $(\mathbf{6 2}, \mathbf{6 3})^{[24]}$ yield enantioenriched alkyl- and selenylcyclopropane products. To the best of our knowledge, this represents the first enantioselective synthesis of selenyl cyclopropanes. Basic and electron-rich heteroaromatics can be installed through telescoped cross-coupling with the corresponding bromides or lithiated heterocycles ${ }^{[25]}$ (see 64-66). Taking advantage of the capacity of NHPI esters as acyl donors, ${ }^{[15]}$ we added Grignard nucleophiles to produce the corresponding alkyl- and arylketones 67-69. We also developed 
a

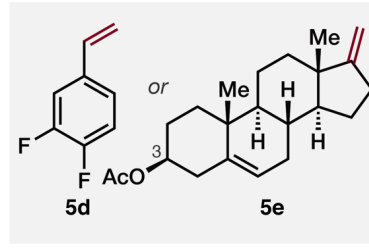

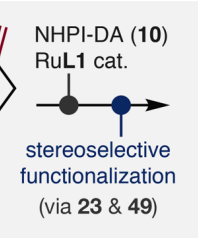

b

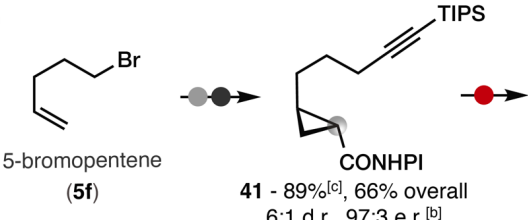
6:1 d.r., $97: 3$ e.r. ${ }^{[b]}$

c<smiles>C=CC[C@H](N)C(=O)O</smiles><smiles>COCCO</smiles>
$69 \%$ overall, $8: 1$ d.r.

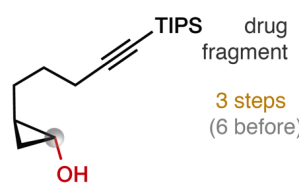

$76-60 \%, 9: 1$ d.r., 95:5 e.r. ${ }^{[b]}$ for grazoprevir (Merck)

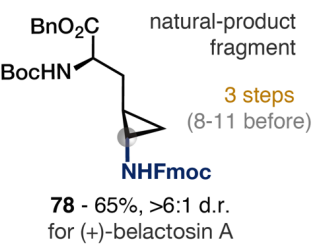

\section{d}

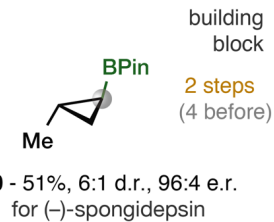

$(5 \mathrm{~h})$

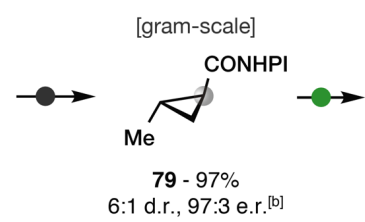

e

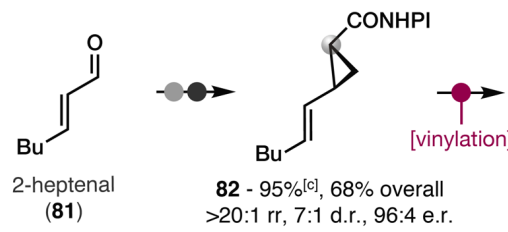

Scheme 4. Synthesis of unrelated enantioenriched functionalized cyclopropanes via redox-active intermediates. See SI for experimental details. ${ }^{[32]}$ [a]C-3 acetate cleaved. ${ }^{[b]}$ Absolute configuration confirmed. ${ }^{[c]}$ Yield of the cyclopropanation step. Yields are calculated on the isolated product. Bpin $=$ boronic acid pinacol ester, 3-Quin = quinolin-3-yl; 3-Py = pyridin-3-yl, 2-Thiop = thiophen-2-yl, Cbz = benzyloxycarbonyl, Teoc $=(2-($ trimethylsilyl)ethoxycarbonyl), Alloc $=$ allyloxycarbonyl, Fmoc $=9$-fluorenylmethyloxycarbonyl .

a direct Curtius amination protocol towards cyclopropylamines $\mathbf{7 0 - 7 5}$, including the ticagrelor ${ }^{\circledR}$ fragments $\mathbf{7 0 - 7 4} .^{[26]}$ It is important to highlight that the synthesis of products $56-75$ did not require neither an individual synthesis of suitable carbene precursors, nor custom catalysts to accommodate their diverse functionalities. These results illustrate the unique versatility of enantioenriched redox-active cyclopropane carboxylates, now accesible in one-step from feedstock alkenes using NHPI-DA (13a).

With these results in hand, we explored the valorization of simple feedstocks in the synthesis of relevant chiral cyclopropanes (Scheme 4b-d). As such, 5-bromopentene (5f) can be transformed in a three-step process into the enantioenriched cyclopropanol fragment $\mathbf{7 6}$ of the drug

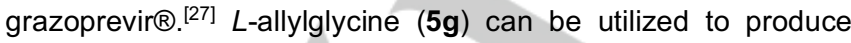
(via 77) the cyclopropylamine fragment 78 of the bioactive natural product (+)-belactosin A (Scheme 4c). ${ }^{[28]}$ Moreover, propene gas $(\mathbf{5 h})$, the simplest pro-chiral aliphatic olefin, leads efficiently on a gram-scale to 79 en-route to the enantiopure cyclopropylboronate building block $\mathbf{8 0}$, used in the total synthesis of (-)-spongidepsin (Scheme 4d). ${ }^{[29]}$ After olefination of the inexpensive 2-heptenal (81; Scheme $4 \mathrm{e})$, the resulting 1,3-diene, is cyclopropanated to yield 82 and further vinylated using a telescoped Zweifel process $\left.{ }^{[25 b}, 30\right]$ into the divinylcyclopropane natural product (-)-dictyopterene A (83). ${ }^{[31]}$ To put these results in perspective, it is important to highlight that the synthesis of the diverse cyclopropane fragments in commercial drugs $(\mathbf{7 0 - 7 4 , 7 6 )}$, natural products $(\mathbf{7 8 , 8 0})$, and the shortest total synthesis of $\mathbf{8 3}$ invariably required 2-3 steps from feedstock materials, utilizing a single strategy, carbene precursor (13a) and catalyst (RuL1). If step count is used to illustrate the tactical value of this new scheme, previous strategies for the asymmetric synthesis of products 70 $\mathbf{7 4 , 7 6 , 7 8 , 8 0 , 8 3}$ required at least twice as many, and up to 15 steps (see Scheme 4) using custom methods, materials, and reagents. The 2-step enantioselective formal transfer of carbenes 6-12 is unprecedented to the best of our knowledge. The current momentum of redox-active ester chemistry and the rich reactivity of carbene transfer suggest future synthetic upgrades of this technology, some of which are underway in our laboratories.

In summary, NHPI-DA (13a) is a practical, safe, and crystalline methine precursor that provides a comprehensive solution to the enantioselective formal transfer of various functionalized methylidenes. This reagent (i) renders the synthesis of custom and unstable carbene reagents unnecessary, (ii) simplifies the optimization of the asymmetric carbene transfer step, and (iii) enables the synthesis of targets with distinct functionalities with the same enantioselectivity and conceptual approach. The unexpected synergistic effect between the geminal redox-active ester and carbene functions enables the general asymmetric cyclopropanation of challenging aliphatic alkenes. Moreover, the NHPI ester function has the unique capacity to promote both acyl-transfer and radical derivatization reactions that enhance the synthetic utility of the resulting enantioenriched cyclopropane intermediates. NHPI-DA (13a) provides the foundation for further asymmetric assembly of 
ubiquitous carbon stereocenters from single carbon units: their simplest retrosynthetic chirons.

\section{Acknowledgements}

The authors are grateful to $\mathrm{Dr} \mathrm{E}$. Martínez de Castro for valuable technical developments and Prof. H. Waldmann, Prof. P. S. Baran and Prof. F. Rodríguez for fruitful discussions. We are indebted to the personnel of AstraZeneca Gothenburg and Stockholm University for unrestricted support. Funds from the Knut and Alice Wallenberg Foundation (KAW2016.0153), and the European Research Council (714737) are gratefully acknowledged. M.M.-M. thanks the Generalitat Valenciana and the European Social Fund for a postdoctoral contract. M.E.M. is a fellow of the AstraZeneca postdoc programme.

\section{Conflict of interest}

A priority patent application has been filed (1850940-6), where A.M., M.M-M., M.C. and E.M.-C. are listed as inventors.

Keywords: Cyclopropane $\cdot$ Enantioselective catalysis $・$ Redoxactive $\cdot$ Diazocompound $\cdot$ Aliphatic Alkene

[1] (a) A. de Meijere, S. I. Kozhushkov, H. Schill, Chem. Rev. 2006, 106 4926-4996; (b) I. W. Davies, L. Gerena, L. Castonguay, C. H Senanayake, R. D. Larsen, T. R. Verhoeven, P. J. Reider, Chem. Commun. 1996, 1753-1754; (c) T. T. Talele, J. Med. Chem. 2016, 59, 8712-8756; (d) D. Y. K. Chen, R. H. Pouwer, J. A. Richard, Chem. Soc Rev. 2012, 41, 4631; (e) C. Ebner, E. M. Carreira, Chem. Rev. 2017, 117, 11651-11679.

[2] Selected reviews: (a) H. Lebel, J. F. Marcoux, C. Molinaro, A. B. Charette, Chem. Rev. 2003, 103, 977; (b) H. Pellissier, Tetrahedron 2008, 64, 7041-7095; (c) G. Bartoli, G. Bencivenni, R. Dalpozzo, Synthesis 2014, 46, 979-1029, 1051 pp; (d) H. Pellissier, A. Lattanzi, R. Dalpozzo, in Asymmetric Synthesis of Three-Membered Rings, WileyVCH Verlag GmbH \& Co. KGaA, 2017, 1-204; (e) H. M. L. Davies, E. G. Antoulinakis, Org. React. 2001, 57, 1-32; (f) M. P. Doyle, D. C. Forbes Chem. Rev. 1998, 98, 911-935.

[3] Selected examples: (a) H. Y. Kim, A. E. Lurain, P. García-García, P. J. Carroll, P. J. Walsh, J. Am. Chem. Soc. 2005, 127, 13138-13139; (b) P. Valenta, P. J. Carroll, P. J. Walsh, J. Am. Chem. Soc. 2010, 132, 14179-14190.

[4] (a) A. B. Charette, H. Juteau, J. Am. Chem. Soc. 1994, 116, 26512652; (b) M.-C. Lacasse, C. Poulard, A. B. Charette, J. Am. Chem. Soc. 2005, 127, 12440-12441; (c) L. E. Zimmer, A. B. Charette, J. Am. Chem. Soc. 2009, 131, 15624-15626; (d) S. Taillemaud, N. Diercxsens, A. Gagnon, A. B. Charette, Angew. Chem., Int. Ed. 2015, 54, 1410814112.

[5] (a) M. Rubina, M. Rubin, V. Gevorgyan, J. Am. Chem. Soc. 2003, 125, 7198-7199; (b) X. Liu, J. M. Fox, J. Am. Chem. Soc. 2006, 128, 56005601; (c) A. Parra, L. Amenós, M. Guisán-Ceinos, A. López, J. L. García Ruano, M. Tortosa, J. Am. Chem. Soc. 2014, 136, 1583315836; (d) L. Dian, I. Marek, Chem. Rev. 2018, 118, 8415-8434.

[6] (a) C. D. Papageorgiou, M. A. C. d. Dios, S. V. Ley, M. J. Gaunt, Angew. Chem., Int. Ed. 2004, 43, 4641-4644; (b) R. K. Kunz, D. W. C MacMillan, J. Am. Chem. Soc. 2005, 127, 3240-3241; (c) T. den Hartog, A. Rudolph, B. Maciá, A. J. Minnaard, B. L. Feringa, J. Am. Chem. Soc. 2010, 132, 14349-14351; (d) J. Carreras, A. Caballero, P. J. Perez, Angew. Chem., Int. Ed. 2018, 57, 2334-2338; (e) J. E. A Luithle, J. Pietruszka, J. Org. Chem. 1999, 64, 8287-8297.

[7] (a) J. i. Ito, S. Ujiie, H. Nishiyama, Chem. Eur. J. 2010, 16, 4986-4990; (b) H. Suematsu, S. Kanchiku, T. Uchida, T. Katsuki, J. Am. Chem.
Soc. 2008, 130, 10327-10337; (c) S. Zhu, X. Xu, J. A. Perman, X. P Zhang, J. Am. Chem. Soc. 2010, 132, 12796-12799; (d) M. M. C. Lo, G. C. Fu, J. Am. Chem. Soc. 1998, 120, 10270-10271; (e) D. A. Evans, K. A. Woerpel, M. M. Hinman, M. M. Faul, J. Am. Chem. Soc. 1991, 113, 726-728; (f) R. E. Lowenthal, A. Abiko, S. Masamune, Tetrahedron Lett. 1990, 31, 6005-6008; (g) H. M. L. Davies, P. R. Bruzinski, D. H. Lake N. Kong, M. J. Fall, J. Am. Chem. Soc. 1996, 118, 6897-6907; (h) X Xu, Y. Wang, X. Cui, L. Wojtas, X. P. Zhang, Chem. Sci. 2017, 8, 43474351; (i) V. N. G. Lindsay, C. Nicolas, A. B. Charette, J. Am. Chem Soc. 2011, 133, 8972-8981; (j) H. Nishiyama, Y. Itoh, H. Matsumoto, S.-B. Park, K. Itoh J. Am. Chem. Soc. 1994, 116, 2223-2224; (k) H. M. L. Davies, P. R. Bruzinski, D. H. Lake, N. Kong, M. J. Fall J. Am. Chem. Soc. 1996, 118, 6897-6907; (I) H. M. L. Davies, P. R. Bruzinski; M. J. Fal Tetrahedron Letters 1996, 37, 4133-4136; (m) M. P. Doyle, et al., J. Am. Chem. Soc. 1995, 117, 5763-5775.

[8] (a) P. S. Coelho, E. M. Brustad, A. Kannan, F. H. Arnold, Science 2013, 339, 307; (b) H. M. Key, P. Dydio, D. S. Clark, J. F. Hartwig, Nature 2016, 534, 534; (c) H. M. Key, P. Dydio, Z. Liu, J. Y. E. Rha, A Nazarenko, V. Seyedkazemi, D. S. Clark, J. F. Hartwig, ACS Cent. Sci. 2017, 3, 302-308; (d) A. M. Knight, S. B. J. Kan, R. D. Lewis, O. F. Brandenberg, K. Chen, F. H. Arnold, ACS Cent. Sci. 2018, 4, 372-377.

[9] For our recent work with aliphatic alkenes see: (a) J. Otero-Fraga, S. Suárez-Pantiga, M. Montesinos-Magraner, D. Rhein, A. Mendoza, Angew. Chem., Int. Ed. 2017, 56, 12962-12966; (b) J. Otero-Fraga, M Montesinos-Magraner, A. Mendoza, Synthesis 2017, 49, 802-809.

[10] J. R. Coombs, J. P. Morken, Angew. Chem., Int. Ed. 2016, 55, 26362649

[11] (a) P. Rulliere, G. Benoit, E. M. D. Allouche, A. B. Charette, Angew. Chem., Int. Ed. 2018, 5777-5782; (b) A. Greb, J.-S. Poh, S. Greed, C Battilocchio, P. Pasau, D. C. Blakemore, S. V. Ley, Angew. Chem., Int. Ed. 2017, 56, 16602-16605; (c) P. R. Schreiner, H. P. Reisenauer, D Ley, D. Gerbig, C.-H. Wu, W. D. Allen, Science 2011, 332, 1300; (d) A. K. Eckhardt, P. R. Schreiner, Angew. Chem., Int. Ed. 2018; (e) G. Benoit, A. B. Charette, J. Am. Chem. Soc. 2017, 139, 1364-1367; (f) C C. Silveira, A. L. Braga, G. L. Fiorin, Synth. Commun. 1994, 24, 2075 2080; (g) J. Barluenga, N. Quiñones, M. Tomás-Gamasa, M.-P. Cabal, Eur. J. Org. Chem. 2012, 2012, 2312-2317; (h) B. Herle, P. M. Holstein, A. M. Echavarren, ACS Catal. 2017, 7, 3668-3675.

[12] (a) M. Rubina, M. Rubin, V. Gevorgyan, J. Am. Chem. Soc. 2004, 126, 3688-3689; (b) L. Dian, D. S. Muller, I. Marek, Angew. Chem., Int. Ed. 2017, 56, 6783-6787; (c) C. Zhong, S. Kunii, Y. Kosaka, M. Sawamura, H. Ito, J. Am. Chem. Soc. 2010, 132, 11440-11442; (d) L. R. Mills, L. M. Barrera Arbelaez, S. A. L. Rousseaux, J. Am. Chem. Soc. 2017, 139, $11357-11360$

[13] (a) J. W. Lehmann, D. J. Blair, M. D. Burke, Nat. Rev. Chem. 2018, 2 0115; (b) K. Colas, R. Martín-Montero, A. Mendoza, Angew. Chem., Int. Ed. 2017, 56, 16042-16046; (c) K. Colas, A. Mendoza, Synlett 2018, 14, 1329-1333.

[14] (a) Z. Wang, A. G. Herraiz, A. M. del Hoyo, M. G. Suero, Nature 2018 , 554, 86; (b) C. Schnaars, M. Hennum, T. Bonge-Hansen, J. Org. Chem. 2013, 78, 7488-7497.

[15] G. H. L. Nefkens, G. I. Tesser, J. Am. Chem. Soc. 1961, 83, 1263 1263.

[16] (a) K. Okada, K. Okamoto, N. Morita, K. Okubo, M. Oda, J. Am. Chem. Soc. 1991, 113, 9401-9402; (b) J. Cornella, et al., J. Am. Chem. Soc. 2016, 138, 2174-2177; (c) K. M. M. Huihui, et al., J. Am. Chem. Soc. 2016, 138, 5016-5019; (d) T. Qin, J. Cornella, C. Li, L. R. Malins, J. T. Edwards, S. Kawamura, B. D. Maxwell, M. D. Eastgate, P. S. Baran, Science 2016, 352, 801; (e) F. Toriyama, J. Cornella, L. Wimmer, T.-G. Chen, D. D. Dixon, G. Creech, P. S. Baran, J. Am. Chem. Soc. 2016 138, 11132-11135; (f) J. T. Edwards, et al., Nature 2017, 545, 213; (g) T. Qin, et al., Angew. Chem., Int. Ed. 2017, 56, 260-265; (h) J. M. Smith, et al., Angew. Chem., Int. Ed. 2017, 11906-11910; (i) A. Tlahuext-Aca, R. A. Garza-Sanchez, F. Glorius, Angew. Chem., Int. Ed. 2017, 3708-3711; (j) D. Wang, N. Zhu, P. Chen, Z. Lin, G. Liu, J. Am. Chem. Soc. 2017, 139, 15632-15635; (k) S. Ni, et al., Angew. Chem. Int. Ed. 2018, 14560-14565; (I) T. G. Chen, et al., Nature 2018, 560, 350-354; (m) X. G. Liu, C. J. Zhou, E. Lin, X. L. Han, S. S. Zhang, Q. Li, H. Wang, Angew. Chem., Int. Ed. 2018, 13096-13100; (n) R. S. J. 
Proctor, H. J. Davis, R. J. Phipps, Science 2018, 360, 419-422; (o) C R. Jamison, L. E. Overman, Acc. Chem. Res. 2016, 49, 1578-1586.

[17] (a) Y. Jin, H. Yang, H. Fu, Org. Lett. 2016, 18, 6400-6403; (b) W. Zhao, R. P. Wurz, J. C. Peters, G. C. Fu, J. Am. Chem. Soc. 2017, 139 12153-12156; (c) R. Mao, A. Frey, J. Balon, X. Hu, Nat. Catal. 2018, 1 120-126.

[18] R. Mao, J. Balon, X. Hu, Angew. Chem., Int. Ed. 2018, 13624-13628.

[19] (a) L. Candish, M. Teders, F. Glorius, J. Am. Chem. Soc. 2017, 139, 7440-7443; (b) A. Fawcett, J. Pradeilles, Y. Wang, T. Mutsuga, E. L. Myers, V. K. Aggarwal, Science 2017, 357, 283; (c) C. Li, et al., Science 2017, eaam7355; (d) J. Wang, M. Shang, H. Lundberg, K. S. Feu, S. J. Hecker, T. Qin, D. G. Blackmond, P. S. Baran, ACS Catal. 2018, 9537-9542.

[20] For alternative divergent cyclopropane syntheses, see for example: (a) S. J. Chawner, M. J. Cases-Thomas, J. A. Bull, Eur. J. Org. Chem. 2017, 2017, 5015-5024; (b) C. W. Liskey, J. F. Hartwig, J. Am. Chem. Soc. 2013, 135, 3375-3378.

[21] With the notable exception of aliphatic olefins, specific conditions and custom diazocompounds have been developed in combination with RuL1: (a) S. Chanthamath, K. Phomkeona, K. Shibatomi, S. Iwasa Chem. Commun. 2012, 48, 7750; (b) A. M. Abu-Elfotoh, K Phomkeona, K. Shibatomi, S. Iwasa, Angew. Chem., Int. Ed. 2010, 49 8439; (c) S. Chanthamath, D. T. Nguyen, K. Shibatomi, S. Iwasa, Org. Lett. 2013, 15, 772; (d) S. Chanthamath, H. W. Chua, S. Kimura, K Shibatomi, S. Iwasa, Org. Lett. 2014, 16, 3408; (e) Y. Nakagawa, S. Chanthamath, K. Shibatomi, S. Iwasa, Org. Lett. 2015, 17, 2792; (f) S. Chanthamath, S. Iwasa, Acc. Chem. Res. 2016, 49, 2080-2090. Cyclopropanation of a single aliphatic olefin (5 equiv) with a diazophosphonate: (g) S. Chanthamath, S. Ozaki, K. Shibatomi, S. Iwasa, Org. Lett. 2014, 16, 3012;

[22] NHPI-DA (13a) has only been prepared in low yield and never used in carbene transfer reactions: (a) I. M. Sakhautdinov, S. N. Lakeev, I. G Halikov, M. F. Abdullin, F. Z. Galin, Bashk. Khim. Zh. 2004, 11, 32-35.
More stable aryl- and alkyl-substituted analogues have been used as diazoacyl-donnors: (b) M. Ma, L. Peng, C. Li, X. Zhang, J. Wang J. Am. Chem. Soc. 2005, 127, 15016. (c) T. Hashimoto, H. Miyamoto, Y. Naganawa, K. Maruoka, J. Am. Chem. Soc. 2009, 131, 11280. (d) T. Hashimoto, H. Nakatsu, K. Yamamoto, K. Maruoka J. Am. Chem. Soc. 2011, 133, 9730.

[23] Mayr, H. Mayr's database of reactivity parameters http://www.cup.Imu.de/oc/mayr/reaktionsdatenbank/.

[24] M. Jiang, H. Yang, H. Fu, Org. Lett. 2016, 18, 1968-1971.

[25] (a) M. Odachowski, A. Bonet, S. Essafi, P. Conti-Ramsden, J. N. Harvey, D. Leonori, V. K. Aggarwal, J. Am. Chem. Soc. 2016, 138, 9521-9532; (b) C. Sandford, V. K. Aggarwal, Chem. Commun. 2017, 53, 5481-5494.

[26] H. Zhang, J. Liu, L. Zhang, L. Kong, H. Yao, H. Sun, Bioorg. Med. Chem. Lett. 2012, 22, 3598-3602.

[27] F. Xu, et al., Org. Lett. 2017, 19, 5880-5883.

[28] O. V. Larionov, A. de Meijere, Org. Lett. 2004, 6, 2153-2156.

[29] H. Lin, L. Tian, I. J. Krauss, J. Am. Chem. Soc. 2015, 137, 1317613182.

[30] R. J. Armstrong, W. Niwetmarin, V. K. Aggarwal, Org. Lett. 2017, 19, 2762-2765

[31] (a) E. Hohn, J. Paleček, J. Pietruszka, Synlett 2008, 2008, 971-974; (b) R. E. Moore, J. A. Pettus, J. Mistysyn, J. Org. Chem. 1974, 39, $2201-$ 2207.

[32] CCDC 1851523 (13a), 1851521 (22), 1851524 (32), 1851525 (41), 1851522 (49) contain the supplementary crystallographic data for this paper. These data can be obtained free of charge from The Cambridge Crystallographic Data Centre. Raw data for this article can be downloaded from Zenodo DOI:10.5281/zenodo.2542646 and DOI:10.5281/zenodo.2542759. 


\section{COMMUNICATION}

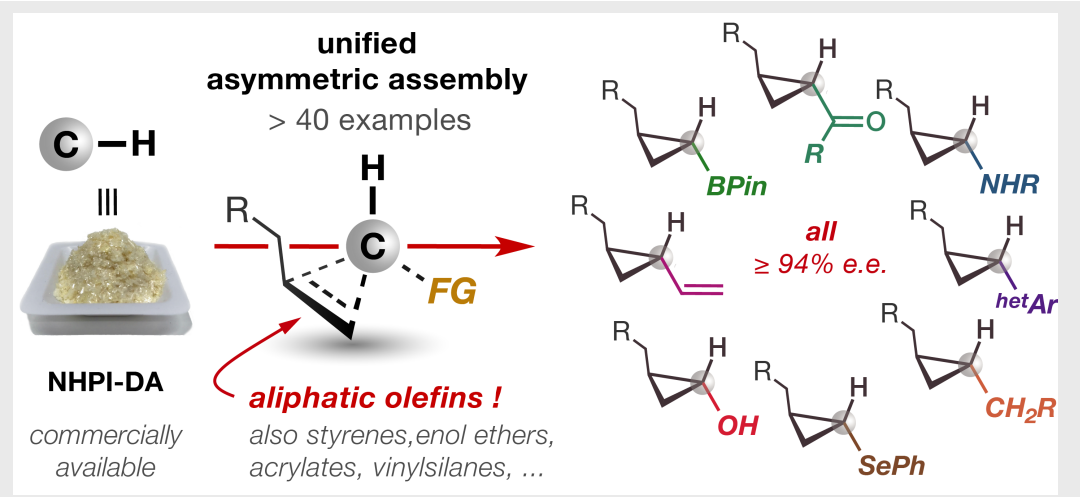

Marc Montesinos-Magraner, ${ }^{[a] \ddagger}$ Matteo Costantini, ${ }^{[a] \pm}$ Rodrigo RamírezContreras, ${ }^{[a]}$ Michael E. Muratore, ${ }^{[b]}$ Magnus J. Johansson ${ }^{[b]}$ and Abraham Mendoza*[a]

Page No. - Page No.

General Cyclopropane Assembly via Enantioselective Transfer of a RedoxActive Carbene to Aliphatic Olefins

The asymmetric synthesis of cyclopropanes is currently designed using specific strategies that depend on the materials available and the final functionality of each target. In this communication, we present a comprehensive approach that engages simple feedstocks, including aliphatic olefins, with a single redox-active carbene precursor (NHPI-DA) that acts as a universal source of a chiral $\mathrm{C}-\mathrm{H}$ unit. 